\title{
Article
}

\section{A kiloparsec-scale nuclear stellar disk in the milky way as a possible explanation of the high velocity peaks in the galactic bulge}

Debattista, Victor P, Ness, Melissa, Earp, Samuel W. F. and Cole, David R.

Available at http://clok.uclan.ac.uk/12573/

Debattista, Victor P ORCID: 0000-0001-7902-0116, Ness, Melissa, Earp, Samuel W. F. and Cole, David R. (2015) A kiloparsec-scale nuclear stellar disk in the milky way as a possible explanation of the high velocity peaks in the galactic bulge. The Astrophysical Journal, 812 (1). L16. ISSN 2041-8213

It is advisable to refer to the publisher's version if you intend to cite from the work. http://dx.doi.org/10.1088/2041-8205/812/1/L16

For more information about UCLan's research in this area go to http://www.uclan.ac.uk/researchgroups/ and search for <name of research Group>.

For information about Research generally at UCLan please go to http://www.uclan.ac.uk/research/

All outputs in CLoK are protected by Intellectual Property Rights law, including Copyright law. Copyright, IPR and Moral Rights for the works on this site are retained by the individual authors and/or other copyright owners. Terms and conditions for use of this material are defined in the policies page. 


\title{
A KILOPARSEC-SCALE NUCLEAR STELLAR DISK IN THE MILKY WAY AS A POSSIBLE EXPLANATION OF THE HIGH VELOCITY PEAKS IN THE GALACTIC BULGE
}

\author{
Victor P. Debattista ${ }^{1}$, Melissa Ness ${ }^{2}$, Samuel W. F. Earp ${ }^{1}$, and David R. Cole ${ }^{1}$ \\ ${ }^{1}$ Jeremiah Horrocks Institute, University of Central Lancashire, Preston PR1 2HE, UK \\ ${ }_{2}^{2}$ Max-Planck-Institut für Astronomie, Königstuhl 17, D-69117 Heidelberg, Germany \\ Received 2015 July 5; accepted 2015 September 25; published 2015 October 9
}

\begin{abstract}
The Apache Point Observatory Galactic Evolution Experiment has measured the stellar velocities of red giant stars in the inner Milky Way. We confirm that the line of sight velocity distributions (LOSVDs) in the mid-plane exhibit a second peak at high velocities, whereas those at $|b|=2^{\circ}$ do not. We use a high resolution simulation of a barred galaxy, which crucially includes gas and star formation, to guide our interpretation of the LOSVDs. We show that the data are fully consistent with the presence of a thin, rapidly rotating, nuclear disk extending to $\sim 1 \mathrm{kpc}$. This nuclear disk is orientated perpendicular to the bar and is likely to be composed of stars on $\mathrm{x} 2$ orbits. The gas in the simulation is able to fall onto such orbits, leading to stars populating an orthogonal disk.
\end{abstract}

Key words: Galaxy: bulge - Galaxy: evolution - Galaxy: formation - Galaxy: kinematics and dynamics Galaxy: stellar content

\section{INTRODUCTION}

Detections of high Galactic standard-of-rest velocity $\left(V_{\mathrm{GSR}}\right)$ peaks in the Apache Point Observatory Galactic Evolution Experiment (APOGEE) commissioning data were reported by Nidever et al. (2012) across all fields at $4^{\circ} \leqslant l \leqslant 14^{\circ}$ and $-2^{\circ}$ $\leqslant b \leqslant 2^{\circ}$. Nidever et al. (2012) interpreted the high- $V_{\mathrm{GSR}}$ peaks as being due to stars in the Galactic bar. However, the peaks are not statistically significant in a number of fields ( $\mathrm{Li}$ et al. 2014) and no high- $V_{\mathrm{GSR}}$ peaks were found at negative longitudes in the Bulge Radial Velocity Assay (BRAVA), at $b \sim=-4^{\circ}$ (Kunder et al. 2012). Additionally, no high- $V_{\mathrm{GSR}}$ peaks can be found in pure $\mathrm{N}$-body models (Li et al. 2014). Molloy et al. (2015) demonstrated that resonant (2:1 and higher order) orbits, viewed on their own, were able to generate high- $V_{\mathrm{GSR}}$ peaks. Aumer \& Schönrich (2015) proposed that such resonant orbits are populated by young stars recently trapped by the bar; they argued that the APOGEE selection function is biased toward such young stars.

Bars have been implicated in building large gas reservoirs at the centers of galaxies, fuelling high star formation rates there. As in other barred galaxies, the Milky Way's (MW) bar funnels gas inwards (Binney et al. 1991; Fux 1999; Weiner \& Sellwood 1999). This gas gives rise to structures such as the Central Molecular Zone (CMZ), spanning $-1^{\circ} \lesssim l \lesssim 1.5$. The CMZ contains 5-10 $\times 10^{7} M_{\odot}$ of molecular gas (Bally et al. 1987; Güsten 1989), driving a star formation rate of $\sim 0.14 M_{\odot} \mathrm{yr}^{-1}$ (Wardle \& Yusef-Zadeh 2008). A molecular gas disk extends across $|l|<6^{\circ}$ and $|b|<1.6$ (Boyce \& Cohen 1994; Dame \& Thaddeus 1994). Liszt \& Burton (1980) and Ferrière et al. (2007) interpreted the observed molecular, atomic and ionized gas outside the CMZ to Galactic longitude $|l| \sim 10^{\circ}$ as a (tilted) disk with semimajor axis of radius $\sim 1.4 \mathrm{kpc}$ with a hole at its center. In external galaxies, star formation in nuclear rings builds nuclear disks (Kormendy \& Kennicutt 2004). In this Letter we demonstrate that the high- $V_{\mathrm{GSR}}$ peaks in the line of sight velocity distributions (LOSVDs) are consistent with the presence of a nuclear disk in the MW.

\section{SIMULATION}

Here we use a high resolution simulation, with gas and star formation, which develops a bar, driving gas to the center and forming a stellar nuclear disk (Cole et al. 2014), to derive the kinematic signatures of such a disk. We use these to guide our interpretation of the APOGEE Data Release 12 (Alam et al. 2015) stellar velocity data for the inner MW. While the simulation was not designed to match the MW, Cole et al. (2014) showed that the nuclear disk that it forms is qualitatively similar to those in external galaxies.

The simulation was evolved with the $\mathrm{N}$-body+smoothed particle hydrodynamics code GASOLINE (Wadsley et al. 2004). The galaxy forms out of gas cooling off a hot corona in pressure equilibrium within a dark matter halo of virial mass $M_{200}=9 \times 10^{11} M_{\odot}$. Both the dark matter halo and the initial gas corona are represented by $5 \times 10^{6}$ particles. As the gas cools and reaches high density, star formation is triggered. Star particles then provide feedback via winds from massive stars, and types Ia and II supernovae (Stinson et al. 2006). Gas particles all have initial mass of $2.7 \times 10^{4} M_{\odot}$ and star particles are spawned from gas with $35 \%$ of this mass. This high mass resolution allows us to use a high star formation threshold of $100 \mathrm{~cm}^{-3}$ for the gas (Governato et al. 2010). By the end of the simulation the galaxy has a stellar mass of $6.5 \times 10^{10} M_{\odot}$ in $\sim 1.1 \times 10^{7}$ particles. This large number of star particles provides a very fine sampling of the mass distribution at the center of the model. Further details of the simulation are provided in Cole et al. (2014).

The bar forms at around $4 \mathrm{Gyr}$. After $6 \mathrm{Gyr}$ a prominent nuclear disk starts to form which, by $10 \mathrm{Gyr}$, has a semimajor axis of $1.5 \mathrm{kpc}$. The nuclear disk is perpendicular to the bar and its stellar streaming is perpendicular to the bar's. At $10 \mathrm{Gyr}$ the nuclear disk in the simulation is quite massive and is thus unlikely to match any nuclear disk in the MW. Therefore here we consider the model at two earlier times: at $t_{1}=6 \mathrm{Gyr}$, before the nuclear disk forms, and at $t_{2}=7.5 \mathrm{Gyr}$ when a strong nuclear disk is established. Aside from the nuclear disk becoming more massive and the bar growing longer, the model at $10 \mathrm{Gyr}$ is not qualitatively different from at $t_{2}$. 


\subsection{Scaling to the MW and Viewing Perspective}

In order to compare to the MW, we rescale the model in both size and velocity. Size rescaling is accomplished by matching the size of the bar to that of the MW. Between $t_{1}$ and $t_{2}$ the average size of the bar in the simulation, as measured from the radius at which the phase of the $m=2$ Fourier moment deviates from a constant by more than $10^{\circ}$ (Aguerri et al. 2003), is $2.1 \mathrm{kpc}$. Assuming that the MW's bar has a semimajor axis of $3.5 \mathrm{kpc}$ (Gerhard 2002), we scale all coordinates by a factor of 1.67 . (Scaling to the more up-todate bar size of Wegg et al. 2015, $5 \mathrm{kpc}$, leads to a nuclear disk which is much too large; because we seek a closer nuclear disk size match, we scale to the older bar size, but this is not to imply that the real MW bar semimajor axis is closer to $3.5 \mathrm{kpc}$ than $5 \mathrm{kpc}$.) The velocity scale factor is obtained by a leastsquares fit to the line of sight velocity dispersion of the model to Abundances and Radial velocity Galactic Origins Survey (Ness et al. 2013) data for all stars within Galactocentric radius $R_{\mathrm{GC}}<3.5 \mathrm{kpc}$ at $b=5^{\circ}, 7.5$ and $10^{\circ}$ across $|l|<15^{\circ}$. We obtain a velocity scaling factor of 0.48 . While these scalings lead to a model of roughly the right size and rotational velocity we stress that the model still does not match the MW and we only use it to qualitatively predict the expected trends in the MW, not their magnitude or precise location.

We assume that the Sun is $8 \mathrm{kpc}$ from the Galactic Center, and place the observer at $y=-8 \mathrm{kpc}$. We orient the bar at $27^{\circ}$ to the line of sight (Wegg \& Gerhard 2013). Since we compare our model with APOGEE (Alam et al. 2015) data, which targets bright red giant stars, we adopt a uniform selection function for star particles at $2 \mathrm{kpc} \leqslant R_{\mathrm{s}} \leqslant 10 \mathrm{kpc}$, where $R_{\mathrm{s}}$ is the distance from the Sun (Schultheis et al. 2014; Hayden et al. 2015). Reducing the maximum $R_{\mathrm{S}}$ to $8 \mathrm{kpc}$ does not significantly alter our conclusions. We use an opening angle of 0.5 for each LOSVD, to match the size of the smallest APOGEE bulge fields. The (off-plane) line of sight with the least particles contains over 2800 star particles while the best sampled (mid-plane) field has over 57,000 star particles; thus the shapes of the model LOSVDs are well determined. The top row of Figure 1 shows the model's surface density distribution.

\subsection{Line of Sight Velocity Distributions}

Viewing the model from the solar perspective, we measure the distribution of line of sight velocities in the Galactocentric restframe, $V_{\mathrm{GSR}}$. Figure 1 shows the LOSVDs for various linesof-sight (indicated in the top row) in the mid-plane $\left(b=0^{\circ}\right.$, second row) and off-plane ( $b=2^{\circ}$, third row). At $t_{1}$ each LOSVD at $l \leqslant 12^{\circ}$ has a single peak, both in the mid-plane and off the plane. The LOSVDs have a shoulder to high $V_{\mathrm{GSR}}$, which Li et al. (2014) showed is produced by stars at large distances seen close to tangentially. The peak in $V_{\mathrm{GSR}}$ moves to larger velocities with increasing $l$, but remains well below the Galaxy's circular velocity. By $t_{2}$ the LOSVDs at $l=8^{\circ}$ and $l=10^{\circ}$ have developed a second, high- $V_{\mathrm{GSR}}$ peak. This peak is more prominent than the low- $V_{\mathrm{GSR}}$ peak, due to the model's very vigorous star formation in the nuclear disk, roughly ten times higher than in the MW for the corresponding region. This very high star formation rate quickly leads to a relatively massive nuclear disk; thus the relative amplitudes of the lowand high- $V_{\mathrm{GSR}}$ peaks are not predictions of the model. Indeed if we reduce the weight of star particles younger than $1 \mathrm{Gyr}$ by a factor of 5, to compensate for the high star formation rate of the model, then the high- $V_{\mathrm{GSR}}$ peaks become smaller than the main peaks, as seen in Figure 1. The distribution around the high$V_{\mathrm{GSR}}$ peak is narrower (i.e., cooler) than that around the main peak and is skewed toward low $V_{\mathrm{GSR}}$. Interior to $l=8^{\circ}$, the LOSVDs are broadened relative to those at $t_{1}$, but no high- $V_{\mathrm{GSR}}$ peak is evident. At $l \geqslant 14^{\circ}$ no high- $V_{\mathrm{GSR}}$ peak is present in the mid-plane, indicating that the structure responsible for the feature does not extend this far. The off-plane and mid-plane LOSVDs are not substantially different at $t_{1}$, aside from the mid-plane hosting more stars at $V_{\mathrm{GSR}} \geqslant 100 \mathrm{~km} \mathrm{~s}^{-1}$. At $t_{2}$ the high- $V_{\mathrm{GSR}}$ peaks, which dominate the mid-plane, are entirely absent in the $b=2^{\circ}$ LOSVDs. Therefore the presence of a nuclear disk is only evident in the mid-plane. As in the MW, outside the nuclear disk, the off-plane LOSVDs at $(l, b)=\left(14^{\circ}\right.$, $2^{\circ}$ ) also contain a statistically significant high- $V_{\mathrm{GSR}}$ peak/ shoulder, but this is also present at $t_{1}$, and is not related to the nuclear disk. Thus the kinematic signatures of a nuclear disk are (1) a second, high- $V_{\mathrm{GSR}}$ peak at roughly the circular velocity, (2) which is absent a few degrees off the mid-plane, (3) is kinematically cooler than the low- $V_{\mathrm{GSR}}$ peak, and (4) is skewed toward low $V_{\mathrm{GSR}}$.

\subsection{LOSVD Stacking}

The top row of Figure 1 shows color-coded maps of the average $V_{\mathrm{GSR}},\left\langle V_{\mathrm{GSR}}\right\rangle$; the peak velocities at orbit tangent points manifest as the characteristic "winged" pattern of the $\left\langle V_{\mathrm{GSR}}\right\rangle$ fields. Although the two $\left\langle V_{\mathrm{GSR}}\right\rangle$ maps show the model before and after the nuclear disk forms, they are not very different, indicating that the formation of the nuclear disk does not lead to a wholesale change of the galaxy as much as populating new parts of its phase space. At the low longitudes of the nuclear disk, large $\left\langle V_{\mathrm{GSR}}\right\rangle$ occurs only close to the galactic center while at other radii $\left\langle V_{\mathrm{GSR}}\right\rangle$ is smaller.

Even with a survey the size of APOGEE, the number of stars in individual fields is still relatively small, giving a low signalto-noise ratio for any second peak in any one field ( $\mathrm{Li}$ et al. 2014). In order to overcome this difficulty, we note that the $V_{\mathrm{GSR}}$ of the second peak does not change significantly with longitude at $6^{\circ} \leqslant l \leqslant 10^{\circ}$. Therefore by stacking the LOSVDs we can enhance the signal-to-noise ratio of the high- $V_{\mathrm{GSR}}$ peak. Because the main peak is dominated by stars streaming along the bar, and $\left\langle V_{\mathrm{GSR}}\right\rangle$ of these changes with $l$, the main peak in a stacked LOSVD will be quite broad. If we include $l<4^{\circ}$, then the exponentially higher density of disk and bar stars near the center masks out any features at high $V_{\mathrm{GSR}}$. In the bottom panels of Figure 1 we present a stack of the model's LOSVDs at $l=6^{\circ}, 8^{\circ}$ and $10^{\circ}$. As with the individual LOSVDs, a peak at high $V_{\mathrm{GSR}}$ is evident at $t_{2}$ in the mid-plane but is absent at $b=2^{\circ}$. Moreover this second peak is still cooler than the low$V_{\mathrm{GSR}}$ peak, and remains skewed toward it. Thus stacking LOSVDs preserves the kinematic signatures of a nuclear disk, and provides a reliable method for searching for a nuclear disk in the APOGEE data.

\section{APOGEE DATA}

\subsection{Data Selection}

We select APOGEE survey stars in the fields of interest, excluding stars with the STAR BAD flag (corresponding to poor stellar parameter fits) and those flagged as flux and telluric 

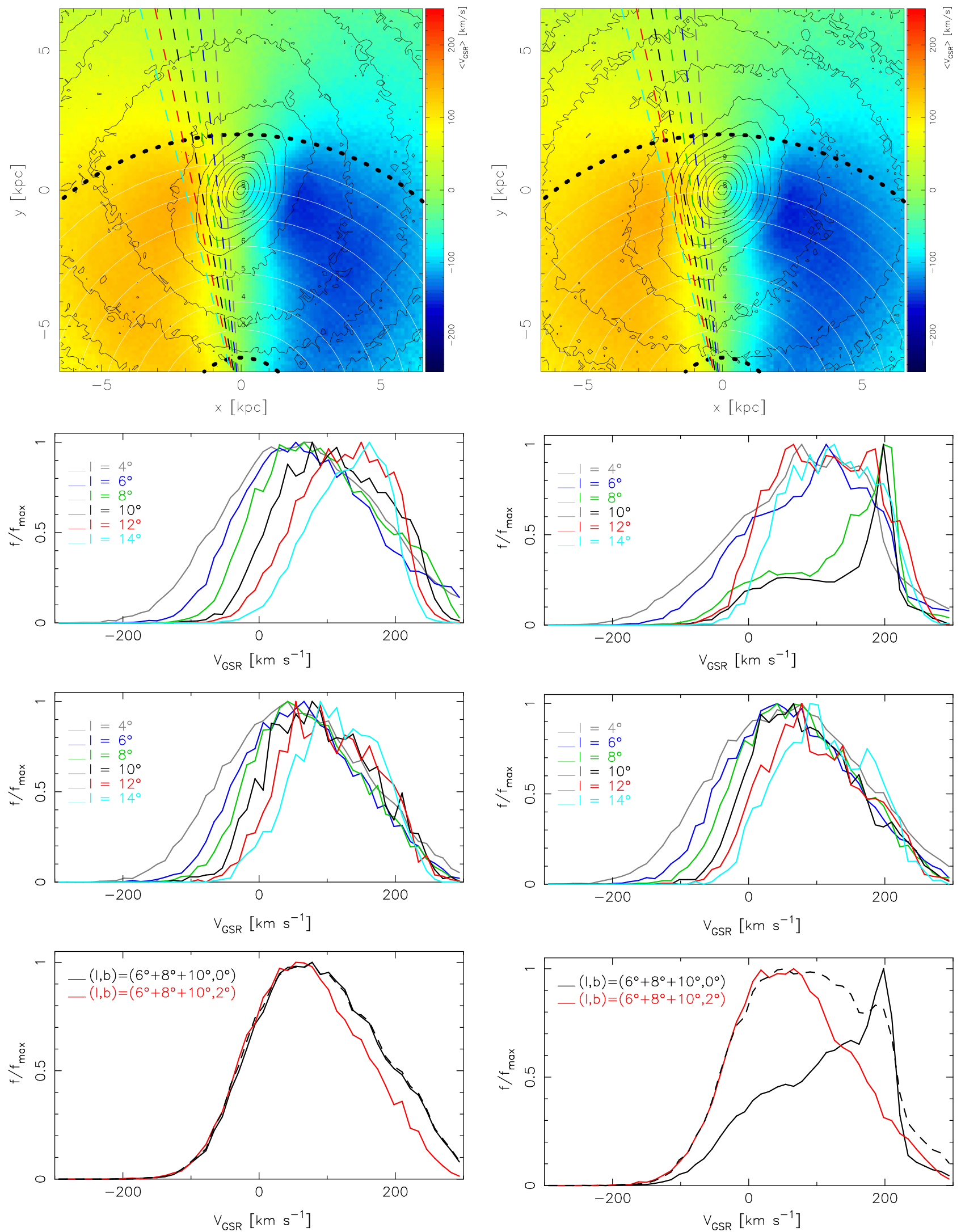

Figure 1. Top row: face-on views of the model: contours indicate the surface density while colors show $\left\langle V_{\mathrm{GSR}}\right\rangle$. The bold dotted circles indicate the radii between which star particles are chosen (the selection function). The dashed lines show longitudes $4^{\circ}-14^{\circ}$ in $2^{\circ}$ steps, color-coded as in the next two rows. Second row: midplane LOSVDs for the different longitudes. Third row: LOSVDs at $b=2^{\circ}$, colored as in the rows above. Bottom row: stacked model LOSVDs from $l=6^{\circ}, l=8^{\circ}$ and $l=10^{\circ}$ in the mid-plane (black) and at $b=2^{\circ}$ (red). The dashed black lines show the effect of reducing the weights of star particles younger than 1 Gyr by a factor of 5 , to compensate for the very high star formation rate in the model. All LOSVDs have been normalized to unit peak. The left panels are at $t_{1}$ while the right ones are at $t_{2}$. 
Table 1

APOGEE Fields used to Construct the Mid-plane and Off-plane Stacks

\begin{tabular}{lcrcc}
\hline \hline Field & $l\left(^{\circ}\right)$ & $b\left(^{\circ}\right)$ & $N_{*}$ & Stack \\
\hline 4336 & 6.0 & 0.0 & 471 & mid-plane \\
4355 & 8.0 & 0.0 & 146 & mid-plane \\
4365 & 5.7 & 2.0 & 387 & off-plane \\
4366 & 5.7 & -2.0 & 424 & off-plane \\
4373 & 7.8 & -2.0 & 154 & off-plane \\
4377 & 7.7 & 2.0 & 149 & off-plane \\
\hline
\end{tabular}

Note. $l$ and $b$ are the Galactic longitude and latitude, respectively, of the field centers. $N_{*}$ is the number of stars selected in each field.

standards. Stars with a velocity scatter between different visits of more than $5 \mathrm{~km} \mathrm{~s}^{-1}$ are also removed. (The same analysis including also stars flagged as STAR BAD, which leads to 763 in the plane and 1401 out of the plane, gives results in agreement with the more conservative cut.)

The small numbers of stars in the APOGEE commissioning data resulted in peaks with low signal-to-noise ratio. We increase the statistical significance of a high- $V_{\mathrm{GSR}}$ peak by stacking the APOGEE DR12 data in the longitude range $6^{\circ} \leqslant l \leqslant 8^{\circ}$ for fields in the mid-plane and off-plane at $|b|=2^{\circ}$ (totalling 617 and 1114 stars, respectively). Table 1 lists the fields stacked together and the number of stars used from each field.

Figure 2 plots these two stacked APOGEE LOSVDs. The mid-plane stack has a clear second peak at $V_{\mathrm{GSR}} \sim 220 \mathrm{~km} \mathrm{~s}^{-1}$, corresponding to roughly the circular velocity of the MW in the bulge region (Sofue et al. 2009). No comparable second peak is visible in the off-plane stacked LOSVD, which is non-Gaussian and skewed toward high $V_{\mathrm{GSR}}$, i.e., it has a shoulder to high $V_{\text {GSR }}$ (Li et al. 2014). A Kolmogorov-Smirnov test shows that the null hypothesis that the mid-plane and off-plane LOSVDs are drawn from the same distribution has a relatively low $p$-value of 0.04 .

We fit two Gaussians to the mid-plane stacked LOSVD in the range $-300 \mathrm{~km} \mathrm{~s}^{-1} \leqslant V_{\mathrm{GSR}} \leqslant 300 \mathrm{~km} \mathrm{~s}^{-1}$, constrained such that the smaller Gaussian contains less than $25 \%$ of the stars (to avoid fitting just the skewed low- $V_{\mathrm{GSR}}$ distribution with two Gaussians). We obtain a low- $V_{\mathrm{GSR}}$ component having mean velocity $\left\langle V_{\mathrm{GSR}}\right\rangle=24 \mathrm{~km} \mathrm{~s}^{-1}$ and standard deviation $\sigma_{\mathrm{GSR}}=57 \mathrm{~km} \mathrm{~s}^{-1}$, while the high- $V_{\mathrm{GSR}}$ component has $\left\langle V_{\mathrm{GSR}}\right\rangle=217 \mathrm{~km} \mathrm{~s}^{-1}$ and $\sigma_{\mathrm{GSR}}=44 \mathrm{~km} \mathrm{~s}^{-1}$, making it cooler than the low- $V_{\mathrm{GSR}}$ component. These two Gaussians are also shown in Figure 2. The velocity distribution at $V_{\mathrm{GSR}} \geqslant 200 \mathrm{~km} \mathrm{~s}^{-1}$ hints at a skewness opposite to that of the main distribution, but the signal-to-noise ratio is still too low for a robust measurement.

The high- $V_{\text {GSR }}$ Gaussian has a significant number of stars associated with it, and is significantly separated from the low$V_{\text {GSR }}$ Gaussian. In order to test the likelihood of such a second peak arising purely from Poisson noise, we perform MonteCarlo tests drawing 617 stars from the off-plane stacked LOSVD. Fitting two Gaussians as before to the resulting LOSVD, we label as $G_{l}$ and $G_{h}$ the low- and high- $V_{\mathrm{GSR}}$ components, respectively. We repeat this procedure 100,000 times, and for each we compute $N_{h} / N_{\text {tot }}$, the ratio of stars in the high- $V_{\mathrm{GSR}}$ component to the total number of stars, and the overlap of the two components, defined as

$$
O=\int G_{l} G_{h} d V_{\mathrm{GSR}}
$$

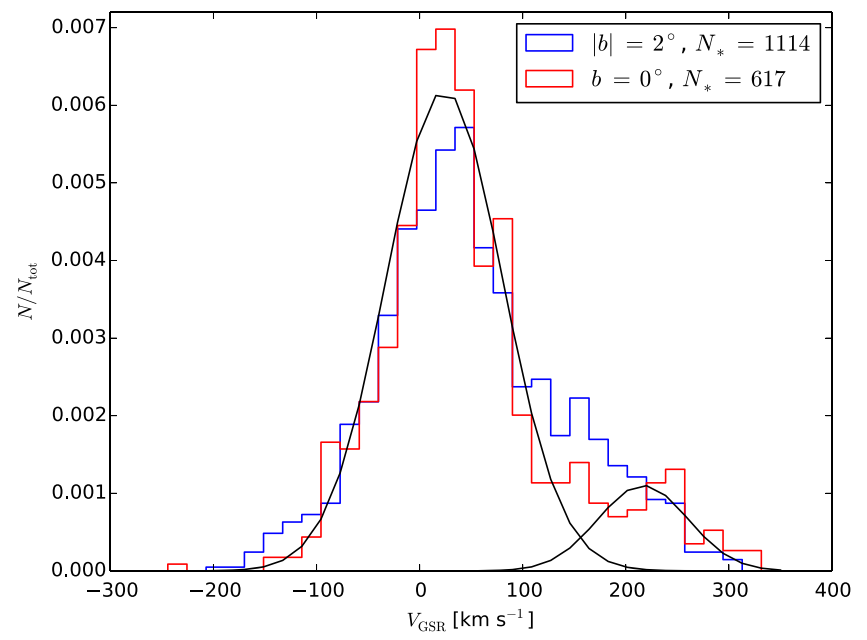

Figure 2. Stacked APOGEE LOSVDs for the mid-plane (red histogram) and at $|b|=2^{\circ}$ (blue histogram). The fields used are listed in Table 1 . The black lines show the two Gaussians fitted to the mid-plane LOSVD.
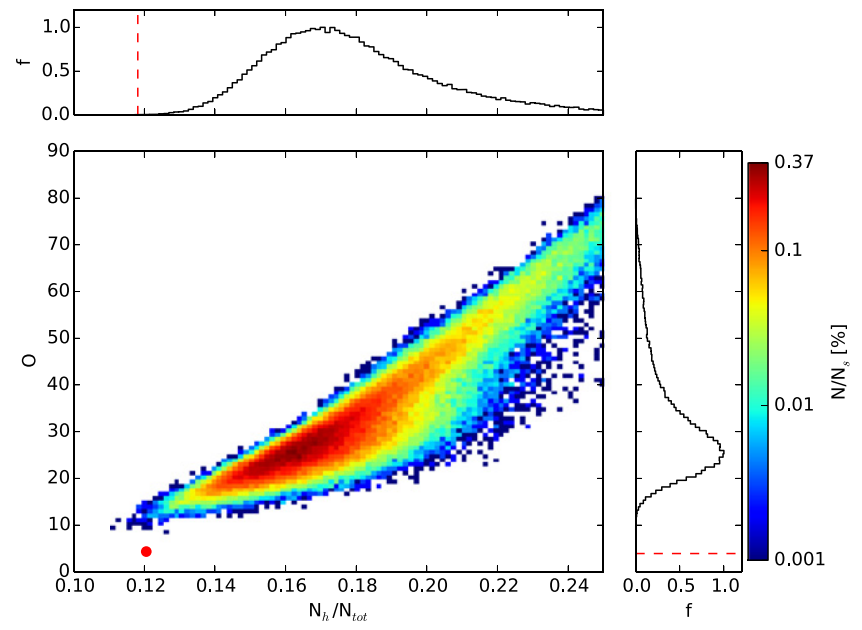

Figure 3. Frequency distribution of properties of double-Gaussian fits for the off-plane APOGEE stacked LOSVD sub-sampled $N_{s}=10^{5}$ times to 628 stars. The side-panels indicate the distributions over the individual variables, normalized to unit peak. The parameters for the fit to the mid-plane APOGEE stack are indicated by the filled red circle. In the side panels, the vertical dashed red lines indicate the values of $N_{h} / N_{\text {tot }}$ and $O$ for the mid-plane stack.

The results are presented in Figure 3; the observed mid-plane stacked LOSVD has $N_{h} / N_{\text {tot }}=0.12$ and $O=4.3$. Only $0.025 \%$ of the Monte-Carlo samples have $N_{h} / N_{\text {tot }} \leqslant 0.12$, while none of them have overlap $O \leqslant 8$, showing that the observed doublepeaked mid-plane stacked LOSVD is highly unlikely to result from Poisson noise. The APOGEE data therefore show a statistically significant double-Gaussian LOSVD in the midplane, the properties of which agree with 3 of the 4 kinematic signatures of a nuclear disk from the simulation. While the signal-to-noise is too low to be sure if the high- $V_{\mathrm{GSR}}$ peak is skewed to low $V_{\mathrm{GSR}}$, the data are suggestive that it is. Therefore a kiloparsec-scale nuclear disk can explain the high- $V_{\mathrm{GSR}}$ peaks in the APOGEE data.

A simple estimate for the nuclear disk mass can be obtained from the fraction of stars in the high- $V_{\mathrm{GSR}}$ component of the double-Gaussian fit to the mid-plane LOSVD. If we conservatively assume that the nuclear disk mass contained within $|z| \leqslant 150 \mathrm{pc}$ and $4^{\circ} \leqslant|l| \leqslant 8^{\circ}$ is $12 \%$ of the total mass of the 
Besançon Galaxy model (Robin et al. 2012) within this volume we obtain a lower limit to the mass of the nuclear disk $\sim 5.8 \times 10^{7} M_{\odot}$.

\section{DISCUSSION}

Attempts to explain the high- $V_{\mathrm{GSR}}$ peak directly via collisionless bar simulations fails (Nidever et al. 2012; Li et al. 2014). However Molloy et al. (2015) demonstrated that resonant, bar-supporting 2:1 x1 (with some mixture of higher order resonance) orbits by themselves can produce second peaks. Subsequently Aumer \& Schönrich (2015) argued that the selection function of APOGEE favors young stars recently trapped into resonant orbits. Their interpretation requires that the stars in the high- $V_{\mathrm{GSR}}$ peaks are younger. The other main $2: 1$ resonant orbit family of bars, the $\mathrm{x} 2$ family, is orientated perpendicular to the bar. This family is generally very poorly populated in the absence of gas (Sparke \& Sellwood 1987; Pfenniger \& Friedli 1991), but when gas is present it is driven inwards by the bar and settles into $\mathrm{x} 2$ orbits (Binney et al. 1991). The gas can then form stars and produce nuclear rings and disks. We propose that the high- $V_{\mathrm{GSR}}$ peak corresponds to a kiloparsec-scale disk composed of stars on $\mathrm{x} 2$ orbits. These orbits are stable and therefore our model does not require that the stars in the high- $V_{\mathrm{GSR}}$ peak are young.

Nuclear disks are known in many external galaxies (Scorza \& van den Bosch 1998; Zasov \& Moiseev 1999; Pizzella et al. 2002; Emsellem et al. 2004; Krajnović et al. 2008; Ledo et al. 2010); the presence of one in the MW is therefore not unusual. Nor is the kiloparsec scale unusual as a fraction of the bar size. For instance in NGC 3945 the ratio of semimajor axes of the nuclear disk to bar is $~ 0.15-0.18$ (Erwin \& Sparke 1999; Cole et al. 2014), whereas for the MW this ratio is $\sim 0.2$, if we adopt Wegg et al. (2015)'s $5 \mathrm{kpc}$ bar. The gas ring in the simulation is $\sim 5 \times$ larger than the MW's CMZ, which is coincident with a stellar disk (Launhardt et al. 2002; Schönrich et al. 2015). The large size of the gas ring in the model is a consequence of the still low resolution $(50 \mathrm{pc})$ of our simulation ( $\mathrm{Li}$ et al. 2015; Sormani et al. 2015). This difference implies that the nuclear disk in the MW is not currently forming stars across its full extent.

We anticipate that this proposal will inspire further detailed mapping of the central mid-plane of the MW. We will provide predictions from our model of a kiloparsec-scale nuclear disk elsewhere.

V.P.D. is supported by STFC Consolidated grants \# ST/ J001341/1 and \# ST/M000877/1, while D.R.C. is supported by STFC Consolidated grant \# ST/J001341/1. M.N. is funded by the European Research Council under the European Union's Seventh Framework Programme (FP 7) ERC Grant Agreement \# 321035. The authors thank the ESF GREAT programme for funding which has supported this research. The simulation used in this paper was run at the High Performance Computing Facility of the University of Central Lancashire. We thank the anonymous referee for a very thoughtful report that helped substantially improve this paper. Funding for SDSS-III has been provided by the Alfred P. Sloan Foundation, the Participating Institutions, the National Science Foundation, and the U.S. Department of Energy Office of Science. The SDSS-III web site is http://www.sdss3.org/. SDSS-III is managed by the Astrophysical Research Consortium for the
Participating Institutions of the SDSS-III Collaboration including the University of Arizona, the Brazilian Participation Group, Brookhaven National Laboratory, Carnegie Mellon University, University of Florida, the French Participation Group, the German Participation Group, Harvard University, the Instituto de Astrofisica de Canarias, the Michigan State/ Notre Dame/JINA Participation Group, Johns Hopkins University, Lawrence Berkeley National Laboratory, Max Planck Institute for Astrophysics, Max Planck Institute for Extraterrestrial Physics, New Mexico State University, New York University, Ohio State University, Pennsylvania State University, University of Portsmouth, Princeton University, the Spanish Participation Group, University of Tokyo, University of Utah, Vanderbilt University, University of Virginia, University of Washington, and Yale University.

\section{REFERENCES}

Aguerri, J. A. L., Debattista, V. P., \& Corsini, E. M. 2003, MNRAS, 338, 465 Alam, S., Albareti, F. D., Allende Prieto, C., et al. 2015, ApJS, 219, 12 Aumer, M., \& Schönrich, R. 2015, MNRAS, in press (arXiv:1507.00907) Bally, J., Stark, A. A., Wilson, R. W., \& Henkel, C. 1987, ApJS, 65, 13

Binney, J., Gerhard, O. E., Stark, A. A., Bally, J., \& Uchida, K. I. 1991, MNRAS, 252, 210

Boyce, P. J., \& Cohen, R. J. 1994, A\&AS, 107, 563

Cole, D. R., Debattista, V. P., Erwin, P., Earp, S. W. F., \& Roškar, R. 2014, MNRAS, 445, 3352

Dame, T. M., \& Thaddeus, P. 1994, ApJL, 436, L173

Emsellem, E., Cappellari, M., Peletier, R. F., et al. 2004, MNRAS, 352, 721

Erwin, P., \& Sparke, L. S. 1999, ApJL, 521, L37

Ferrière, K., Gillard, W., \& Jean, P. 2007, A\&A, 467, 611

Fux, R. 1999, A\&A, 345, 787

Gerhard, O. 2002, in ASP Conf. Ser. 273, in The Dynamics, Structure \& History of Galaxies: A Workshop in Honour of Professor Ken Freeman, ed. G. S. Da Costa \& H. Jerjen (San Francisco, CA: ASP), 73

Governato, F., Brook, C., Mayer, L., et al. 2010, Natur, 463, 203

Güsten, R. 1989, in IAU Symp. 136, The Center of the Galaxy, ed. M. Morris (Dordrecht: Kluwer Academic), 89

Hayden, M. R., Bovy, J., Holtzman, J. A., et al. 2015, ApJ, 808, 132

Kormendy, J., \& Kennicutt, R. C. 2004, ARA\&A, 42, 603

Krajnović, D., Bacon, R., Cappellari, M., et al. 2008, MNRAS, 390, 93

Kunder, A., Koch, A., Rich, R. M., et al. 2012, AJ, 143, 57

Launhardt, R., Zylka, R., \& Mezger, P. G. 2002, A\&A, 384, 112

Ledo, H. R., Sarzi, M., Dotti, M., Khochfar, S., \& Morelli, L. 2010, MNRAS, 407, 969

Li, Z., Shen, J., \& Kim, W.-T. 2015, ApJ, 806, 150

Li, Z.-Y., Shen, J., Rich, R. M., Kunder, A., \& Mao, S. 2014, ApJL, 785, L17 Liszt, H. S., \& Burton, W. B. 1980, ApJ, 236, 779

Molloy, M., Smith, M. C., Wyn Evans, N., \& Shen, J. 2015, arXiv:1505.04245

Ness, M., Freeman, K., Athanassoula, E., et al. 2013, MNRAS, 432, 2092

Nidever, D. L., Zasowski, G., Majewski, S. R., et al. 2012, ApJL, 755, L25 Pfenniger, D., \& Friedli, D. 1991, A\&A, 252, 75

Pizzella, A., Corsini, E. M., Morelli, L., et al. 2002, ApJ, 573, 131

Robin, A. C., Marshall, D. J., Schultheis, M., \& Reylé, C. 2012, A\&A, 538, A106

Schönrich, R., Aumer, M., \& Sale, S. E. 2015, arXiv:1507.02695

Schultheis, M., Zasowski, G., Allende Prieto, C., et al. 2014, AJ, 148, 24

Scorza, C., \& van den Bosch, F. C. 1998, MNRAS, 300, 469

Sofue, Y., Honma, M., \& Omodaka, T. 2009, PASJ, 61, 227

Sormani, M. C., Binney, J., \& Magorrian, J. 2015, MNRAS, 449, 2421

Sparke, L. S., \& Sellwood, J. A. 1987, MNRAS, 225, 653

Stinson, G., Seth, A., Katz, N., et al. 2006, MNRAS, 373, 1074

Wadsley, J. W., Stadel, J., \& Quinn, T. 2004, NewA, 9, 137

Wardle, M., \& Yusef-Zadeh, F. 2008, ApJL, 683, L37

Wegg, C., \& Gerhard, O. 2013, MNRAS, 435, 1874

Wegg, C., Gerhard, O., \& Portail, M. 2015, MNRAS, 450, 4050

Weiner, B. J., \& Sellwood, J. A. 1999, ApJ, 524, 112

Zasov, A. V., \& Moiseev, A. V. 1999, in IAU Symp. 194, Activity in Galaxies and Related Phenomena, ed. Y. Terzian, E. Khachikian, \& D. Weedman (San Francisco, CA: ASP), 279 